\title{
Mitteilungen
}

Société médicale de la Suisse romande (SMSR)

\section{Le Dr Philippe Eggimann est le nouveau président de la SMSR}

La Société médicale de la Suisse romande (SMSR) a renouvelé son Comité. C'est désormais le Président de la Société vaudoise de médecine (SVM), Philippe Eggimann, qui préside également l'association faîtière romande des sociétés cantonales de médecine.

Le Dr Philippe Eggimann (VD) a été désigné le 4 octobre 2018 en tant que nouveau président de la Société médicale de la Suisse romande (SMSR) pour un mandat de 3 ans, parallèlement à son mandat de président de la Société vaudoise de médecine (SVM) récemment renouvelé pour deux ans. Il succède à cette fonction au Dr Bertrand Jacot-Descombes (GE), qui assurait la présidence ad intérim de la SMSR depuis le décès intervenu en cours de mandat du Dr Pierre-Alain Schneider (GE), en mars 2018. Le Dr Bertrand Jacot-Descombes reste membre du Comité de la SMSR en tant que vice-président.

La composition du nouveau Comité de la SMSR est désormais la suivante. Bureau: Dr Philippe Eggimann (VD) président, Dr Bertrand Jacot-Descombes (GE), vice-président, Dr Remo Osterwalder (JU), vice-président. Autres membres du Comité en tant que présidents des sociétés cantonales romandes: Dr Jean-Marie Michel (FR),

Dr Michel Matter (GE), Dr Claude Schwarz (JU), Dr Walter Gusmini (NE) et Dr Monique Lehky Hagen (VS).

Né en 1960 et exerçant depuis cette année au Département de l'appareil locomoteur (DAL) du CHUV, le Dr Philippe Eggimann est titulaire d'un Diplôme (1985) et d'un Doctorat (1990) de médecine de l'Université de Lausanne, des Diplômes FMH de spécialiste en infectiologie (1999), spécialiste en médecine intensive (1995) et spécialiste en médecine interne (1994). Côté politique professionnelle, le Dr Philippe Eggimann est notamment Président de la Société vaudoise de médecine (SVM) depuis 2016, membre de la Chambre Médicale de la FMH depuis 2012 et de l'Assemblée des délégués de la FMH depuis 2016. Il a rejoint le Comité de la SMSR en 2016.

"Les défis sont nombreux pour la SMSR dans un contexte national délicat», explique le Dr Philippe Eggimann. «En particulier, alors que les coûts de la santé imputables aux médecins se stabilisent dans tous les cantons romands, nous considérons comme tout à fait injustifiées les nouvelles hausses de primes encore récemment annoncées, ainsi que la pression croissante mise sur les prestataires.»

Renseignements complémentaires: Dr Philippe Eggimann, président de la SMSR, 0794394935 www.smsr.ch

40. Sportweltspiele der Medizin und Gesundheit vom 22. bis 29. Juni 2019 in Montenegro

Grösstes Sportereignis für Mediziner und Kollegen aus den gesundheitlichen Berufen

Die Sportweltspiele der Medizin und Gesundheit, auch bekannt als Medigames, feiern ihr 40. Jubiläum vom 22. bis 29. Juni 2019 in Montenegro, genauer im Städtchen Budva an der Adria. Montenegro ist in der Öffentlichkeit noch wenig bekannt und ein angesagtes Reiseziel. Diese Perle der Adria verfügt über ein reiches Natur- und Architekturerbe und ein ausgeglichenes mediterranes Klima.

Seit nun 40 Jahren begeistern die Sportweltspiele jedes Jahr bis zu 2000 sportliche Mediziner, Ärzte, Apotheker und Kollegen aus den gesundheitlichen und pflegenden Berufen, die mit Freunden und Familien aus mehr als 40 Ländern anreisen. Aus dem deutschsprachigen Raum haben bei der Veranstaltung 2018 über ein Drittel Frauen aktiv teilgenommen; die deutschen Teilneh- mer führten den internationalen Medaillenspiegel an.

\section{Sportwettkämpfe ein kommunikatives} Ereignis

Die Wettkämpfe der Sportweltspiele werden in fairer Atmosphäre ausgetragen und bieten den Teilnehmern die Möglichkeit, internationale Freundschaften zu schliessen und in über 20 verschiedenen Sportdisziplinen an den Start zu gehen. Die Sportwettkämpfe werden ausser den Mannschaftssportarten sowie Golf und Schach in sechs Alterskategorien gewertet. Das tägliche gesellige und folkloristische Programm und die allabendlichen Siegerehrungen runden das Sportevent $a b$.

\section{Teilnahmebedingungen klar bestimmt}

Teilnehmen können Medizinerinnen und Mediziner sowie Kollegen aus medizinischen und pflegenden Berufen. Auch Studenten und Auszubildende aus den Fachbereichen können akkreditiert werden. Ein gesundheitliches Attest und Freude am Leistungssport sind Voraussetzungen. Erwünscht ist auch das Entdecken von neuen Sportarten, die alle zum Einheitspreis praktiziert werden können.

Die Sportweltspiele sind wegen der grossen Vielfalt der angebotenen Disziplinen und Startmöglichkeiten für Amateursportler einmalig. Von Tennis über Leichtathletik und Schwimmen, Radrennen und Fussball bis hin zum Orientierungslauf und Segeln reicht die Liste der Disziplinen, bei denen auch Freunde

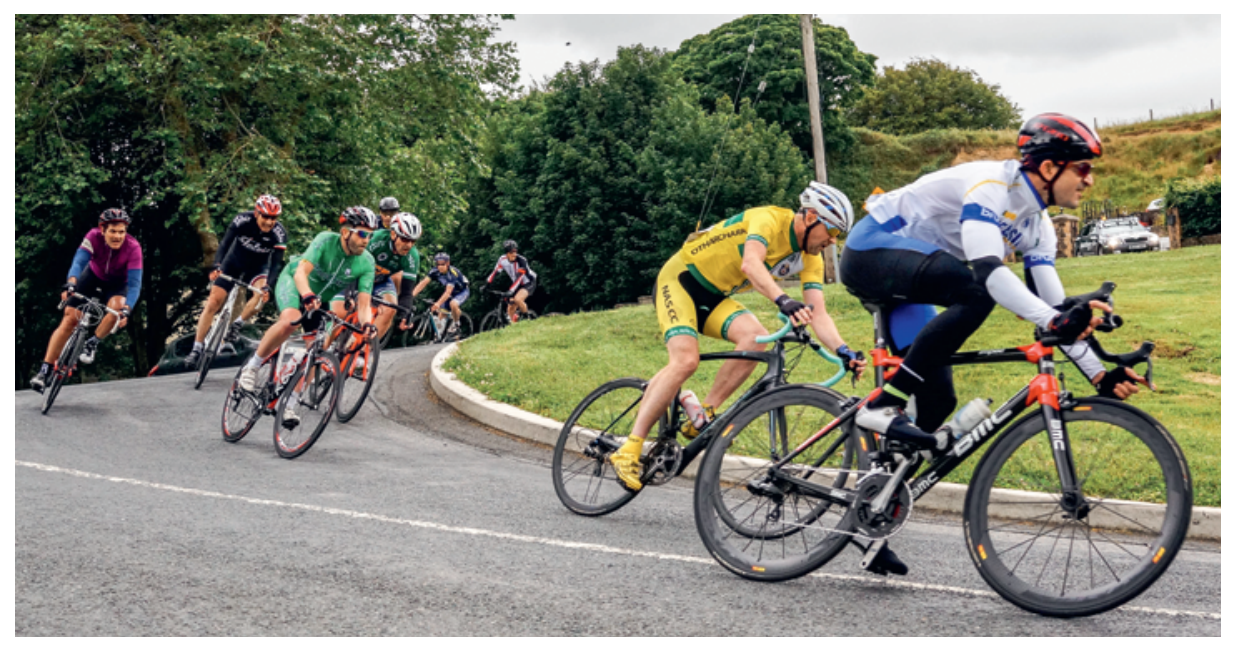

Strassenrennen. 
und Angehörige der Teilnehmer ohne Wertung mitstarten können.

Neben den sportlichen Wettkämpfen bieten die Sportweltspiele einen internationalen Kongress für Sportmedizin und einen Erfahrungsaustausch mit Kollegen aus aller Welt. Alle Vorträge werden auf Englisch und Französisch angeboten. Ein «Posting-Raum» wird ebenfalls zur Verfügung stehen, in dem Teilnehmer ihre Forschungsergebnisse vorstellen können. Abgabedatum für Konferenzbeiträge ist der 15. April 2019. Offizieller Anmeldeschluss zur Sportweltspieleteilnahme ist der 31. Mai 2019, doch der Veranstalter bemüht sich, noch bis kurz vor Beginn Kurzentschlossene zu berücksichtigen.

Die jährlich tournierende Sportveranstaltung wird seit 1995 von der Corporate Sport Organisation (Frankreich) organisiert. Die Teilnahme- und Unterkunftskosten, Teilnahmebedingungen und Anmeldemöglichkeiten stehen online unter www.sportweltspiele.de.

Bildnachweis

www.sportweltspiele.de

\section{PharmaSuisse}

\section{Vor 175 Jahren gegründet:}

der Schweizerische Apothekerverband

Er ist der älteste hiesige Branchenverband: der Schweizerische Apothekerverband. Am 18. Oktober 1843 wurde er in Zürich von 13 Männern gegründet mit dem Zweck, das kollegiale Band unter den Mitgliedern zu fördern. Seither haben sich Frauen ihren Platz erkämpft, die Ausbildung von Apothekern wurde universitär, und ohne Apotheker wäre die medizinische Versorgung von Frau und Herrn Schweizer nicht sichergestellt.

Schon seit Mitte der dreissiger Jahre des 19. Jahrhunderts hatten einige forsche Apotheker versucht, die Pharmazeuten landesweit zu vereinen. Erst ein Deutscher konnte es richten: Theodor Hübschmann importierte das Konzept einer geeinten Apothekerschaft und brachte 13 Männer im damaligen Hotel Krone in Zürich zusammen. Am 18. Oktober 1843, also vor 175 Jahren, gründeten sie den Schweizerischen Apotheker-Verein. Zweck war vor allem Networking. Die noch erhaltene Gründungsurkunde spricht von einem "kollektiven Band", das es zu knüpfen galt.

Apotheken als Keimzellen der Naturwissenschaften

Apotheker waren damals «Handwerker».
Sie sammelten Wissen, betätigten sich als Botaniker oder Chemiker. Rund 30 Jahre nach der Gründung erneuerten und vereinheitlichten sie die Ausbildung, Pharmazie wird universitär. Noch heute absolvieren Apotheker ein 5-jähriges Universitätsstudium, absolvieren danach eine 2- bis 3-jährige Weiterbildung und bilden sich lebenslang fort. Die junge Gesellschaft war aber auch gefordert im Kampf rund um die Abgrenzung zu Ärzten. Die zentrale Frage: Wer hat das nötige Wissen rund um Herstellung, Abgabe und Beratung zu Medikamenten? Heute wissen wir: Wenn es um die ganzheitliche Gesundheitsversorgung der Schweizer Bevölkerung geht, braucht es interprofessionelle Zusammenarbeit. Ebenso erkämpfen mussten sich die Frauen ihren Platz im neuen Verein. 1900 schreiben sich die ersten Studentinnen an den Hochschulen ein, Clara Winnicki erhält als erste Schweizerin ihr Apothekerdiplom. Es ist der Beginn einer starken Feminisierung des Berufs. Heute sind von den 6300 berufstätigen Apothekern gut zwei Drittel Frauen.

\section{Seit 175 Jahren voraus}

Immer wieder haben Apothekerinnen und Apotheker mit Entdeckergeist und offenem Herzen clevere Lösungen für anstehende Herausforderungen im Gesundheitswesen gefunden. Sie geben 1865 die Pharmakopöe für die Schweiz heraus, ein amtliches Arzneibuch, das verbindliche Qualitätsvorschriften für Arzneimittel vorgibt. Sie setzen ihr Wissen in der Armee ein, prägen die industrielle Herstellung von Medikamenten mit und engagieren sich für die Medikamentenversorgung in Spitälern. Heute stellen die 1800 Schweizer Apotheken die Versorgung der Bevölkerung mit Medikamenten sicher. Aber weit mehr: Sie geben Kunden und Patienten Orientierung, begleiten und betreuen sie bei Therapien und helfen mit einfachen Präventionsmassnahmen, die Bevölkerung gesund zu behalten und Kosten im Gesundheitswesen tief zu halten. Kein Wunder, dass die Politik explizit wünscht, dass Apotheker ihre Kompetenzen erweitern - die Anpassung des Medizinalberufegesetzes 2015 und des Heilmittelgesetzes 2016 zeugen davon. Und bereits jetzt testet der Schweizerische Apothekerverband pharmaSuisse Lösungen für morgen, die dazu beitragen, dass chronisch kranke Menschen ihre Therapie durchziehen - und ein Einsparpotential von theoretisch 30 Milliarden Franken für das schweizerische Gesundheitswesen bietet.

Dem Verband sind rund 6300 Einzelmitglieder und 1500 Apotheken angeschlossen. www.pharmaSuisse.org

\section{Interpharma}

\section{Förderung des Tierwohls - Jahresbericht} zum Tierschutz in der Pharmaindustrie

Um die Bedingungen bei Tierversuchen und den Schutz von Labortieren weiter zu verbessern und zu fördern, wurde 2010 von der forschenden Pharmaindustrie der Schweiz die Tierschutzcharta ins Leben gerufen.

Im heute publizierten Jahresbericht informieren die Interpharma-Mitgliedsfirmen über konkrete Forschungsprojekte im Bereich 3R und Alternativmethoden sowie über die Fortschritte zum Wohle des Tieres.

Die forschende Pharmaindustrie erfüllt in der Schweiz die Auflagen einer der weltweit striktesten Tierschutzgesetzgebungen. Die Interpharma-Mitgliedsfirmen sind sich der Verantwortung bewusst und sind basierend auf der im Jahr 2010 geschaffenen Zehnpunktecharta bestrebt, die hohen Standards bei Tierversuchen durch konkrete Projekte laufend weiterzuentwickeln und weltweit Verbesserungen zu implementieren.

\section{Konsequente Umsetzung der 3R-Prinzipien}

Die forschenden pharmazeutischen Firmen richten sich konsequent nach den Leitsätzen der 3R-Tierschutzprinzipien und forschen ständig nach weiteren Verbesserungen mit dem Ziel, die erforderliche Zahl der Tiere zu reduzieren (Reduce), die Belastung der Labortiere auf ein Minimum zu beschränken (Refine) und Tierversuche zu ersetzen (Replace). In der Schweiz stand am Anfang der 3R-Forschung die Stiftung Forschung 3R, die 1987 ins Leben gerufen wurde. Die Stiftung unterstützte die Forschung für bessere Methoden oder Alternativen zu Tierversuchen und wurde von Beginn an durch den Bund und Interpharma paritätisch finanziert. Das neue nationale 3R-Kompetenzzentrum soll diese Entwicklung weiterführen.

Auch auf Firmenebene wird ständig nach Verbesserungen im Sinne des Tierwohls geforscht. Einzelne Mitgliedsfirmen von Interpharma verleihen jährlich 3R-Preise, mit denen sie wegweisende interne Projekte und Erkenntnisse auszeichnen, und unterstützen zahlreiche internationale Forschungsprojekte und -gruppen. Die Suche nach Alternativmethoden lohnt sich für die Tiere und die Firmen, denn Alternativmethoden sind meistens weniger kostenintensiv und liefern besser reproduzierbare Resultate als Tiermodelle. Um einen Einblick in die aktuelle Forschung zu gewähren, werden im 
diesjährigen Jahresbericht einige Beispiele für 3R-Preise und für Alternativmethoden vorgestellt.

\section{Menschliche Organe im Miniaturformat}

Die bislang einzige Möglichkeit, das komplexe Zusammenspiel verschiedener menschlicher Organe abzubilden, ist die Verwendung von Versuchstieren. Aber auch bei Tiermodellen sind die Ergebnisse nicht immer auf den Menschen übertragbar. Deswegen wird in jüngster Zeit sowohl in der Pharmaindustrie als auch in der Akademie stark an der "Organs on a chip»-Technologie geforscht. Mit der neuartigen Technologie können Wirkstoffe an künstlich nachgebauten Mini-Organen getestet werden. Auf diesen dreidimensionalen Organ-Chips finden sich Bedingungen, wie sie im menschlichen Organ anzutreffen sind. Neben der besseren Übertragbarkeit auf den Menschen fördern die Organe auf dem Chip auch die 3R-Prinzipien.

Den vollständigen «Animal Welfare Report 2018» finden Sie auf unserer Website unter www.interpharma.ch in der Rubrik Publi-
kationen/Forschung. Er steht dort als PDF zur Verfügung. Die Druckversion der Broschüre kann auf Deutsch, Französisch und Englisch bezogen werden.

Kontakt:

\section{Sara Käch}

Leiterin Kommunikation Interpharma Tel. 061264 34 14, Mobile 0792081633 E-Mail: sara.kaech[at]interpharma.ch

\section{Aktuelles Thema auf unserer Website} www.saez.ch/de/tour-dhorizon
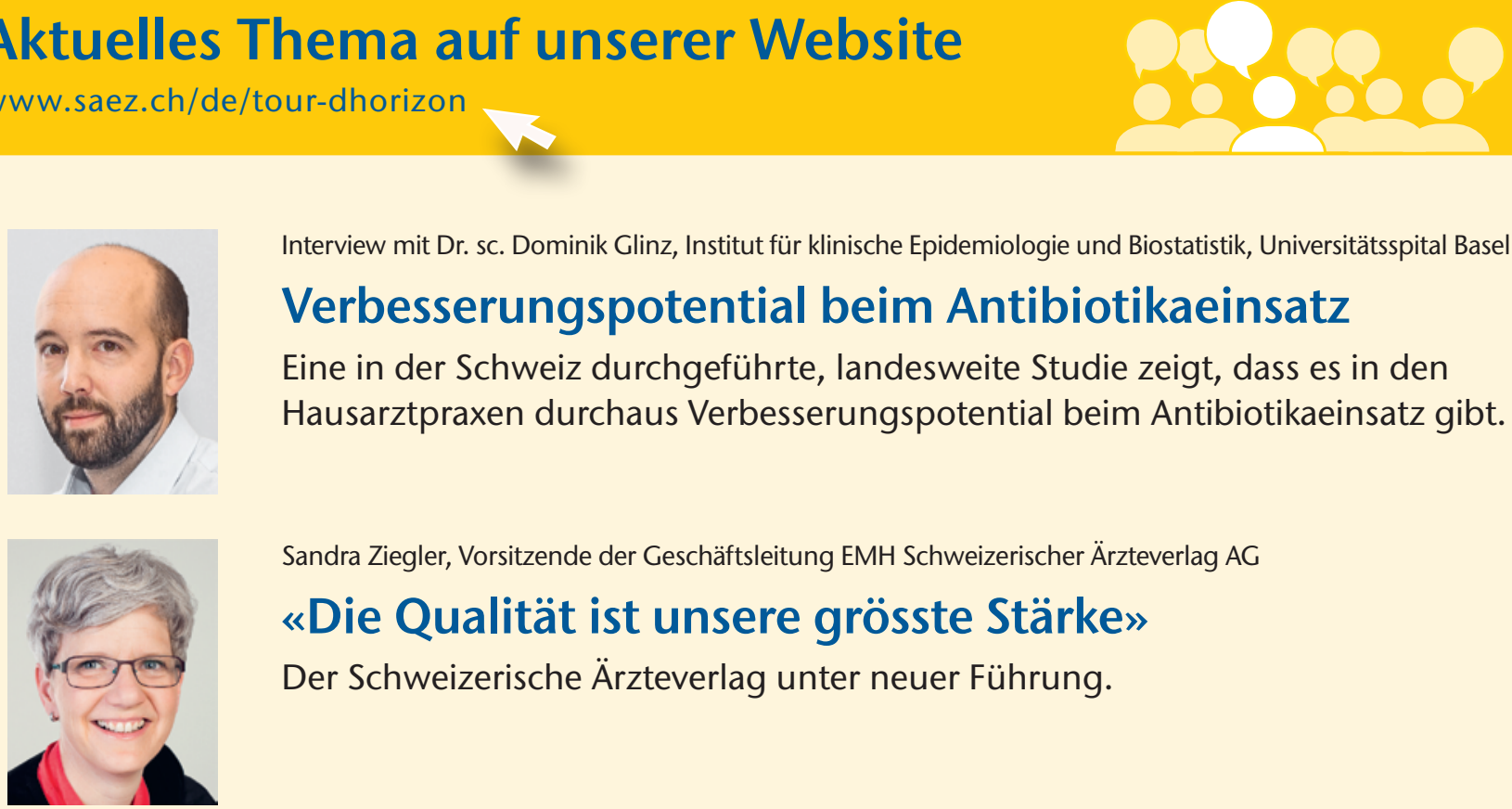

Interview mit Dr. sc. Dominik Glinz, Institut für klinische Epidemiologie und Biostatistik, Universitätsspital Basel

\section{Verbesserungspotential beim Antibiotikaeinsatz}

Eine in der Schweiz durchgeführte, landesweite Studie zeigt, dass es in den Hausarztpraxen durchaus Verbesserungspotential beim Antibiotikaeinsatz gibt.

Sandra Ziegler, Vorsitzende der Geschäftsleitung EMH Schweizerischer Ärzteverlag AG

\section{«Die Qualität ist unsere grösste Stärke»}

Der Schweizerische Ärzteverlag unter neuer Führung. 